\title{
An Empirical Study of Foreign Language Reading Based on Formative Assessment Teaching Model
}

\author{
Mai Tong ${ }^{1, \mathrm{a}}$, Zhao xinxia ${ }^{2, \mathrm{~b}^{*}}$ \\ ${ }^{1}$ School of Foreign Languages, Northwest University for Nationalities, Lanzhou, Gansu, China \\ ${ }^{2}$ School of Foreign Languages, Northwest University for Nationalities, Lanzhou, Gansu, China \\ a422296680@qq.com \\ b*1041054144@qq.com
}

\begin{abstract}
This paper explores the influence of formative assessment based teaching mode on the improvement of students' foreign language reading ability. Through 36-week classroom experiments, it is shown that students' learning motivation and self-reflection consciousness are strengthened. The frequency of students using various reading strategies increases, and their reading scores improve gradually. The students' non-intellectual factors are developed. The research proves that the formative assessment teaching model is helpful to improve students' foreign language reading ability and plays a positive role in promoting college foreign language reading teaching.
\end{abstract}

Keywords: Formative evaluation, Foreign language reading, Curriculum teaching, Autonomous learning ability

\section{基于形成性评价教学模式的外语阅读实证研究}

买通 ${ }^{1,}$ a 赵新霞 ${ }^{2}$, b*

\author{
'西北民族大学外国语学院，兰州，甘肃，中国 \\ 2 西北民族大学外国语学院，兰州，甘肃，中国 \\ a422296680@qq.com \\ b*1041054144@qq.com
}

\section{摘要}

本文探究基于形成性评价教学模式对学生外语阅读能力提升的影响, 通过 36 周课堂实验表明: 学生的学习动机、 自主反思的意识均得到强化; 学生运用各种阅读策略的频次增加, 阅读成绩逐步提升; 学生的非智力因素得到 发展。研究证明, 形成性评价教学模式有助于提高学生的外语阅读能力, 对大学外语阅读教学有积极的推动作 用。

关键词 形成性评价; 外语阅读; 课程教学; 自主学习能力

\section{1.引言}

教学评估是大学外语教学环节中的组成要件, 发 挥着导向性作用。然而, 长久以来形成的标准化考查 模式，造成了“分数至上”的学习取向。考试成绩只能 说明学习者语言知识的片面积累, 而无法全面检测学 习者的语言综合应用能力和非智力因素在学习过程 中的支配性作用。这种终结性评价无法较好地反映学 习全过程, 更不利于高质量外语人才的综合发展与培
养。

1967年, 芝加哥大学斯克里芬教授（Michael Scriven）撰文 “评价方法论”（The Methodology of Evaluation）并首次提出“形成性评价”的概念, 当时这 种评价仅用于改进课程编制的问题。两年之后, 教育 心理学家布卢姆( B.S.Bloom) 将形成性评价推广到 教与学的过程中。他认为“形成性评价是在课程编制、 教学和学习过程中使用的一种系统性评价机制, 对这 三个过程中的任何一个过程都能加以改进 。”自 80 年 
代, 越来越多的学者开始关注形成性评价与终结性评 价的区别，如 Bachman（1981），Brown（1989）, 90年代形成性评估的研究范围涵盖理论、工具、研究 路径以及对教学的影响 (Genesee \& Upshur1996)。

Bell\&Cowie(2001)研究认为,形成性评价具有终 结性评价不具备的重要特征:（1）对教学和学习过程 不间歇地反应性; (2) 具有多层次,有效度的质化和量 化数据来源; (3) 隐形性, 非正式的过程; (4) 教师在 评价中的中坚作用; (5) 与教学过程密不可分。

形成性评价在国内学界的应用始于 20 世纪 80 年 代中期, 研究者针对终结性评价在人才培养中存在的 弊端探讨实施形成性评价的重要性, 多数研究成果局 限于教育学的范畴。直至 21 世纪初, 国内学者在外语 教学领域围绕形成性评价的应用研究才呈现出不断 上涨的趋势, 国内外语教学形成性评价研究成果可分 为三个层面: (1)综述研究: 部分学者围绕理论综述, 梳理国内形成性评价研究的领域、特点、方法与总体 趋势 (吴秀兰2008; 贾军红2013; 袁树厚、束定芳2017); 部分学者通过介绍国外研究最新进展推进该领域向 多视角、跨学科的纵深方向发展(罗凯洲、韩宝成, 2014)。（2）理论研究: 一些学者采用扎根理论和课 堂话语分析的方法, 描述课堂形成性评价目标的动态 变化特征（文秋芳, 2014) ; 一些学者从理论角度构 建形成性评价的效度验证框架, 认为课堂评估的质量 评价体系是效度验证的关键（李清华，2008）。（3） 应用研究: 该领域的研究主要探讨形成性评价在具体 课程教学中的应用 (写作、口语、阅读、翻译等) 以 及不同评价主体反馈能力的差异（季配英，2017）。

中国学界认为, 形成性评价是一种以评价为导向 的课堂活动范式, 要求评价者 (老师、学生) 采用各 种适当的任务 (课堂提问、任务、纸笔测试、档案袋 等), 系统地收集学生信息 (包括学习产品与学习过 程), 并用适当的评价工具（检查表、评分标准等） 对信息进行评价分析与阐述, 再反馈给评价者 (教师、 学生) 用于教与学改进的过程, 以促进学生语言能力 的发展。

\section{2.研究设计}

\section{1. 授课类型}

阿拉伯语阅读2、3是西北民族大学阿拉伯语专业 二年级开设的专业必修课, 周二学时, 授课内容为外 语教学与研究出版社出版的《新编阿拉伯语》第二、 三册的课后阅读部分。根据《高等学校阿拉伯语专业 基础阶段阿拉伯语教学大纲》中对阅读技能的教学要 求, 专业二年级学生应“掌握细读方法, 初步掌握快 读、略读、寻读等阅读方法; 能够在 10 分钟内读完与 课文难度相当的 350 词左右的短文并理解大意。”在大 纲的语言技能表中对阅读技巧罗列出更为细致的要 求, 诸如“通过上下文了解词义、根据文章结构进行 推断、根据标识符号进行推断”等内容。故课程大纲
与教案的设计与实施应当以大纲指导, 将阅读技能的 应用与提升作为教学的重点。

\section{2. 研究目标}

本次研究以西北民族大学外国语学院阿拉伯语 专业2018级的两个教学班为研究对象, 计划于 2019-2020学年展开为期36周的阅读教学实践活动。 其中实验班人数为 20 人, 男生 7 人, 女生 13 人; 控制 班人数为 20 人, 男生 6 人, 女生 14 人, 共计 40 名学生。 笔者对实验班实施基于形成性评价的教学模式, 而控 制班则沿用传统的教学讲授模式。旨在论证基于形成 性教学评价的教学模式是否可以实现以下目标:

(1) 形成性评价教学模式有助于激发学生阅读 兴趣, 强化自我反思的意识。

（2）该模式的实施有助于提升阅读能力，尤其 阅读策略的综合运用能力。

(3) 成绩处于中低水平学生的自信心、态度等 非智力因素的表现有所改观。

\section{3. 研究模型}

基于实验目标, 笔者设计了基于形成性评价教学 模式在外语阅读教学中的应用框架:

每个教学单元划分为阅读前活动、阅读中活动、 阅读后活动三个环节。阅读前活动包括学习目标的共 建和预习任务的发布。学习目标 “共建” 是指教师在 明确教学目标的前提下指导学生建立适合自己的学 习目标, 即学生经过实践活动可以达到的实际目标。 这种设计体现出学科知识结构和学生认知结构的统 一; 阅读中的活动以任务教学为核心。教师根据单元 内容设计阅读任务, 要求学生以小组为单位实施并展 开互评, 教师完成点评与总结, 以实现生生反馈与教 师反馈的结合; 阅读后的活动以课后拓展任务与自我 评价为主。以下是该模型的流程设计图:

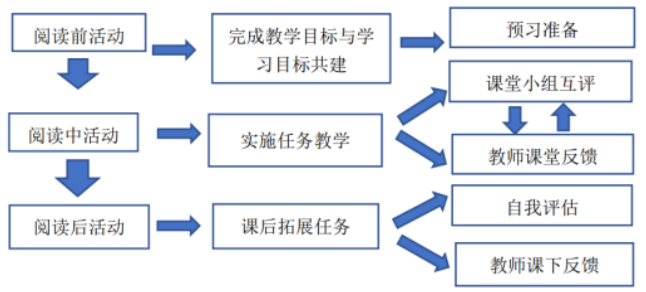

图1 基于生成性评价的外语阅读教学流程设计图

\section{3.研究过程与数据收集}

\section{1. 实验过程}

笔者在36周的教学周期内对实验班实施形成性 评价教学模式, 根据课程大纲中的教学安排, 完成 18 个教学单元。实验班学生完成每周的单元阅读任务后 实施自我评价。教师每月集中检查学生的自评材料 
（自评档案袋）, 不定期展开课下访谈与问卷调查; 控制班按照传统教学模式进行教学, 同样接受阅读任 务, 但在研究期间不获得任何反馈。

\section{2.数据收集}

(1) 阅读能力调查问卷。笔者在学年初与学年 末分别向实验班、控制班学生发放调查问卷。该问卷 设计为三大版块: 第一版块旨在描述学生阿拉伯语阅 读动机的强弱 (共 3 题) 、第二版块统计学生阅读 策略的使用情况（共 14 题）、第三版块分析学生的 自主学习与反思能力 (共 12 题) 。研究结束后, 笔者 累计回收有效问卷 80 套, 通过对比数据结果分析形成 性评价教学模式对提升学生阅读能力的影响。

(2) 阿拉伯语阅读前、后测试卷。笔者在 2019-2020学年第一周和第36周分别对实验班与控制 班实施阅读前、后测。测试内容均选自世界图书出版 公司出版的《阿拉伯语阅读教程（2）》中的文章。 根据测试要求, 学生需在 45 分钟内完成难易度接近、 不同题材的三篇文章。用于比较阅读成绩的变化。

(3) 课下访谈。本学期教学实验前, 笔者根据 学习成绩将实验班学生分为三个层次 (100-85,85-65,65-55), 并分别从中选择两名学生作 为访谈跟踪对象。每个月进行一次访谈, 搜集这6名 同学对基于形成性评价阅读教学的反馈信息, 记录他 们在阅读心理障碍、自信心、意志力等方面的变化。 学期结束后, 笔者将这些反馈做整理汇总。

\section{4.数据结果分析}

\section{1. 调查问卷分析}

笔者在学期初发放的问卷调查结果显示: 两个班 学生在阅读动机上的差异并不明显, 实验班中有强烈 阅读动机的学生比例略低于控制班（分别为 $28.6 \%$ 与 $31.8 \%$ )，不喜欢阅读的学生比例同样低于控制班(分 别为 $12.5 \%$ 与 $14.3 \%)$ 。在阿拉伯语阅读策略使用方面, 实验班与控制班都只有较少比例的学生 (分别为 $13 \%$ 与 $14.2 \%$ ) 会综合使用多种阅读策略（略读、寻读、 查读、生词推测、段落主题句提取、主旨大意概述等), 大部分学生 (分别为 $52.5 \%$ 与 $55 \%$ ) 学生会使用多种 阅读策略中的某一种或两种, 其中使用频率最高的是 略读和寻读策略。在第三板块 (自主学习与反思能力) 中, 两个班基本维持在同一水平, 只有 $16.3 \%$ 的实验 班学生会有意识地通过制定学习计划、学习总结等手
段来强化阅读技能, $83.7 \%$ 的学生较少甚至从未进行 过学习反思。

形成性评价实践结束后的问卷结果表明:实验班 学生在前面提到的三个方面均有明显的改观。其中 $70.6 \%$ 的学生表示喜欢上阅读课,较之前的 $28.6 \%$ 提升 了 $42 \% ; 72.4 \%$ 的学生经过两学期的阅读教学实践, 逐步认识并懂得如何应用多种阅读策略。略读、寻读、 猜测词义占据阅读策略使用频率的前三位; $82.6 \%$ 的 学生开始树立反思的意识, $58 \%$ 的学生会制定阶段性 的阅读计划（单元、星期、月份），完成周期性自我 评价总结及不定期地复习难点与错题。而对比控制班 的前后问卷数据，除了阅读策略综合使用频率有小幅 提升，其他指标基本保持同一水平

\section{2. 学生访谈分析}

教学实践结束后，笔者将 8 次访谈的反馈信息汇 总整理如下：（1）阅读心理障碍在不同成绩水平的 学生中体现出一定的差异: 中低成绩水平学生反映最 多的是由于基础薄弱、缺乏督促带来的自信心缺失; 较高成绩水平学生的心理障碍集中体现在紧张情绪 的影响。（2）经过两个学期的教学实践, 中低成绩 水平的学生得到教师更多的关注。在课堂持续的良性 反馈与课下针对性辅导的影响下, 他们逐步树立了自 信心, 缓解了自卑、消极的情绪。较好成绩水平的学 生经过周期性的训练强化，培养了良好的阅读习惯与 反思能力, 阅读紧张的心理障碍也逐步克服。（3） 访谈对象均表示形成性评价课堂中的参与度提升, 学 生之间产生更多的协作和交流（4）访谈对象均对形 成性评价教学均持肯定的态度。

\section{3. 阅读前后测数据分析}

在阅读前测中, 实验班学生的阅读速度为每分钟 29词，控制班的学生阅读速度为每分钟31词。根据高 等学校阿拉伯语专业基础阶段教学大纲的规定, 学生 的正常阅读速度为每分钟 35 词。可见两班学生的阅读 速度与大纲要求还有一定差距。在阅读后测中, 实验 班学生的阅读的速度为每分钟 41 词, 比控制班的成绩 多出6个单词。可见, 在阅读教学中运用形成性评价 更有利于学生阅读速度的提高。

笔者又利用社会统计学软件 SPSS19.0 对实验班 与控制班阅读成绩进行了全面分析，包括两班阅读前 后测成绩的独立样本 $\mathrm{T}$ 检验、实验班阅读前后测的成 绩的配对样本 $\mathrm{T}$ 检验，数据统计如下：

表 1: 实验班与控制班阅读前测成绩独立样本 $\mathrm{T}$ 检验

\begin{tabular}{|c|c|c|c|c|c|}
\hline & 均值 & 样本量 & 标准差 & $\begin{array}{c}\text { 平均标准误 } \\
\text { 差 }\end{array}$ & 差异显著性 \\
\hline 实验班 & 72.3500 & 20 & 11.979 & 2.67864 & $\begin{array}{c}\mathrm{T}=-.482 \\
\mathrm{P}>0.05\end{array}$ \\
\hline 控制班 & 74.3400 & 20 & 14.1877 & 3.1724 & \\
\hline
\end{tabular}


表 2: 实验班与控制班阅读后测成绩独立样本 $\mathrm{T}$ 检验

\begin{tabular}{|l|l|l|l|l|l|}
\hline & 均值 & 样本量 & 标准差 & 平均标准误差 & 差异显著性 \\
\hline $\begin{array}{l}\text { 实验班前 } \\
\text { 测 }\end{array}$ & 72.3500 & 20 & 11.9792 & 2.67864 & $\begin{array}{l}\mathrm{T}=7.930 \\
\mathrm{P}<0.05\end{array}$ \\
\hline $\begin{array}{l}\text { 实验班后 } \\
\text { 测 }\end{array}$ & 84.250 & 20 & 8.0581 & 1.8018 & \\
\hline
\end{tabular}

表3: 实验班与控制班阅读后测成绩配对样本T检验

\begin{tabular}{|l|l|l|l|l|l|}
\hline & 均值 & 样本量 & 标准差 & 平均标准误差 & 差异显著性 \\
\hline 实验班 & 84.2500 & 20 & 8.0581 & 1.8018 & $\begin{array}{l}\mathrm{T}=3.406 \\
\mathrm{P}=0.02<0.0 \\
5\end{array}$ \\
\hline & & & & & \\
\hline 控制班 & 76.0500 & 20 & 13.8468 & 3.09624 & \\
\hline
\end{tabular}

生评价中, 师生关系会对学生评价产生偏见, 不同的

从表一中可以看到, 控制班的前测成绩平均值略 高于实验班, 但实验班的平均标准误差值低于控制班, 说明分数较为集中, 独立样本 $\mathrm{T}$ 检验结果表明, $\mathrm{t}$ 值为 -0.482, P值大于 0.05 说明两个班的成绩差异并不大; 表二中, 实验班的后测成绩平均值为 84.25 分, 高于 控制班的76.05分, 同时实验班的平均标准误差值依 然低于控制班, 说明实验班阅读成绩普遍提高且分数 较为集中。独立样本 $\mathrm{T}$ 检验结果表明, $\mathrm{t}$ 值为-3.406, 双尾 $\mathrm{T}$ 检验概率为 0.02 , 小于 0.05 显示差异具有显著 意义; 表三中, 实验班“前测”的均值为72.35分, 标准 差为 11.9792 , “后测”的均值为 84.250 分, 标准差为 8.0581 。后测阅读成绩比前测阅读成绩有显著提高 (高出11.9分), 同时因为后测标准差小一些, 分数 较前测集中。配对样本 $\mathrm{T}$ 检验结果表明, $\mathrm{t}$ 值为-7.930, $\mathrm{P}$ 值为 0.000 , 小于 0.05 显示差异具有显著意义, 说明 形成性评价的实施与阅读成绩之间呈正相关的趋势。

\section{5.结论}

经过为期 36 周的形成性评价教学实践, 实验班 学生的自主学习能力增强, 能更好地规划、监控和评 估自己的学习过程; 学生的非智力因素得到发展。在 形成性评价教学实践中, 师生之间的互动反馈、生生 之间交流普遍降低了学生对于阅读的恐惧感与焦虑 情绪。教师在课堂上给予中低成绩水平学生更多的良 性反馈, 使他们的自信心逐步树立起来, 激发出有益 于学习的情感来不断促进学习; 教学质量进一步提升, 教师通过有效反馈了解学生的掌握水平, 从而在教学 目标与实际达成目标之间进行有效调节, 促进教学质 量的提高。多元的评价体系促进了师生之间的有效沟 通, 使教师摆脱唱独角戏的困境。丰富的任务教学形 式有助于提升学生的阅读兴趣, 培养协作能力。形成 性评价教学模式使学生在课前、课上、课后都被调动 起来, 促进了知识的吸收、转化与应用。

虽然形成性评价教学模式对阿拉伯语阅读教学 产生了积极而广泛的影响, 但是在具体的实施操作过 程中仍然暴露不少问题, 主要包括以下几个方面: 师
任课教师难以确保公平、统一的评价标准; 学生互评 环节中, 虽有教师提供的评分原则为参考, 但实际打 分时主观性较强; 缺乏评价的经验与智慧, 容易导致 小组内表现较差的学生丧失积极性, 而不愿继续参与; 自我评估过程中, 学生对自身学习水平认知不足, 容 易出现目标过高或者过低的倾向; 教学进度的调整缺 乏合理性的依据, 仅凭课堂观察难以准确了解不同学 生对知识点的掌握程度; 评估之后缺乏有效的衔接, 教师往往在评估之后发现问题, 但缺乏有效手段进行 定期监督, 使评估仅仅停留在对学情的分析上。针对 于以上问题，需要从以下方面加强:

第一，规范评估标准，完善评估体系。师生、生 生、自评使评价主体多元化, 同时也增加了评价的难 度。师生评价应该建立公平、统一的标准; 学生互评 中的评分标准应当在教师的指导下完成, 要求清晰、 客观、易操作。学生完成评分的同时需要陈述具体的 理由并给出针对性的建议; 学生在自主确立学习目标 时不能脱离实际, 应参考教师面向全体学生制定的教 学目标。以周、月为单位评估目标任务的实际完成度, 然后做阶段性调整。

第二, 拓展教学弹性, 促进良性反馈。课程进度 以课程大纲的规定为准。如果学生对某单元知识点普 遍接受困难, 可以适当放缓授课节奏, 但不能耽误总 体教学任务的完成。对于互评过程中表现较差的学生, 组内成员的评分应当有一定弹性, 同时教师需在课下 给予更多的指导与关注, 以帮助他们树立信心。

第三, 实现有效监督, 保证学习质量。学情的评 估如同一面镜子, 反映出学习过程中的诸多短板, 但 如果没有长期有效的监督, 学生依旧难以走出困境。 教师可以通过课堂问答、课后作业、阶段性测验、课 下访谈等多种方式督促学生掌握单元任务。同时定期 检查学生的学习档案与自评材料, 并将此列入平时成 绩的考核当中。

形成性评价的应用符合我国教学的发展趋势, 有 利于教育理念及教学模式的转变, 对于当前教学改革 
无疑将具有重大的指导意义。从以上问题可以看出, 教师评价、同伴评价的公平性、自我评价的有效性、 评价激励与纠错机制的完善将成为深化形成性评价 改革的重要内容, 促使其成为大学阿拉伯语阅读教学 中质量监控、提升教学效果的有效环节。

\section{项目基金}

本文为甘肃省 2020-2022 年高等院校外语教学研 究项目《促进民族院校外语课程混合式教学效果的途 径研究》(GSSKB20-26)的阶段性成果之一. 本文为西 北民族大学国家一流专业建设项目的阶段性成果之 一。

\section{REFERENCES}

[1] Bloom.B.S. (1981). Education to Improve Learning. McGraw-Hill Publishing, NewYork.

[2] Bloom. B.S. (1988) Educational evaluation. Translated by Qiu Yuan, Wang Gang, East China Normal University Press, Shanghai.

[3] Luo Shaoqian. (2003) A Study on Classroom Formative Evaluation of English Teaching. Foreign Language Teaching and Research Press, Beijing.

[4] Shi.L, (1996). Curriculum Theory: Fundamentals, Principles and Problems of Curriculum. Education
Science Press, Beijing.

[5] BLACK P, WILLIAM D. (1988) Inside the black box: Raising standards through classroom assessment. J. Phi DeltaKappan, 2: 139-148.

[6] Fan Weiwei, (2013), Self-evaluation of Chinese College Students' Intercultural Competence.J. Foreign Languages in China, 6 :53-59.

[7] Wang Jie. (2016) Formative Evaluation of College English Autonomous Learning.J. Journal of East China University of Science and Technology (Social Sciences), 2:122-132.

[8] Hansen, M. \& Hansen, J (2000) A cloud evaluation model for formative evaluation of English teaching. J. Journal of Foreign Language Teaching, 9:71-75.

[9] Yang Manzhen, Liu Jianda. (2019) Research on College English Teaching Practice Based on Formative Evaluation. J. Audio-visual Teaching of Foreign Languages. 6: 97-102

[10] Minqiang T, Tongzhe S. (2009) The construction and thinking of the evaluation system of network multimedia foreign language teaching. $\mathrm{J}$. Audio-visual Teaching of Foreign Languages, 5: 65- 69. 